\title{
Research on Game Decision Structure Model of Enterprise Alliance
}

\author{
Siyuan Chen \\ Department of Foundation, Xi'an Siyuan University, Xi'An 710038, China \\ xsucsy@163.com
}

Keywords: Symbiosis Mechanism, Game Theory, Cooperative Game, Decision-making Mechanism

Abstract. research of enterprise's cooperative decision-making is an important part of the theory of enterprise strategic management. Enterprise's cooperative decision-making can be further perfected and enrich the contents of the theory of enterprise strategic management. In this paper, the dynamic model of enterprise strategic decision making is established by using evolutionary game theory. The key factors affecting the alliance are analyzed. The relationship between the key factors and the relationship between the key factors and the alliance enterprise are analyzed.

\section{Introduction}

The external factors that influence the stability of the alliance are the interaction between the alliance and the external environment. The change of enterprise's competitive position is influenced by the change of environment. The non symmetric symbiosis is a dynamic environment in which the external environment is the basis of the alliance movement. The stability of the symbiotic Alliance under consideration must be considered [1]. Such as natural environment, political environment, economic environment, market environment and other changes will cause the effective operation of the alliance cooperation, resulting in a reduction of the overall revenue of the alliance, even cause the collapse of the alliance, as shown in figure.1 [2].

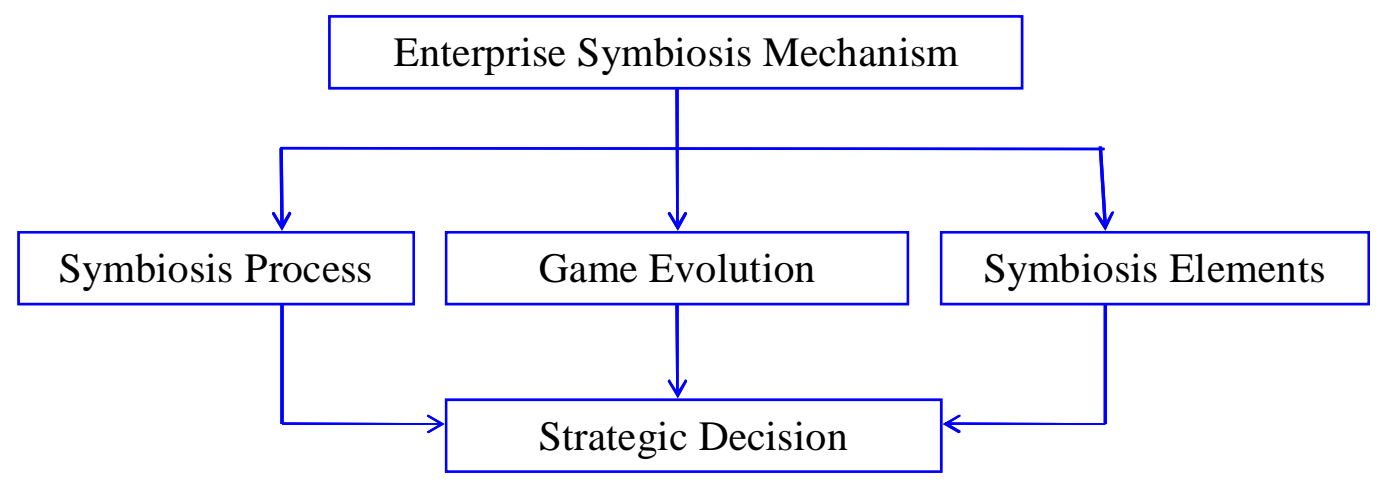

Figure. 1 The framework of this research

Based on the premise of limited rationality, the key factors affecting the symbiotic alliance are obtained, and the equilibrium and stability of the evolutionary game model are analyzed.

\section{Basic theory of Enterprise Alliance Cooperative Game}

With the classical game theory and ecological theory, the theory of negative traditional game theory, which is based on the limited rational people's point of view, is to overcome the difficulties of rational hypothesis and multiple equilibrium in new classical economics and the theory of limited rationality [3]. The dynamic representation of the dynamic differential equation of the dynamic differential equation of the dynamic differential equation of the dynamic differential equation, which is used in the individual or population, is used for reference:

$$
\frac{d x}{d t}=x\left(\mu_{y}-\bar{\mu}\right)
$$


In the formula: $x$ is the ratio of the type of trust type, $\mu_{y}$ is the expected return of the agreement strategy, $\bar{\mu}$ is the mean of all game parties, $d x / d t$ is the ratio of the type of trust type with time [4]. Dynamic differential equation is proportional to the ratio of the game to the type of trust type, and it is proportional to the expected profit of the type of game and the average profit of the game. The external environment, the limitations of their own conditions, and the complexity of the decision making problems themselves make it difficult to achieve complete rationality. On the basis of this, the alliance's choice strategy is adjusted by time, and the pursuit of dynamic equilibrium and stability, as shown in figure.2.

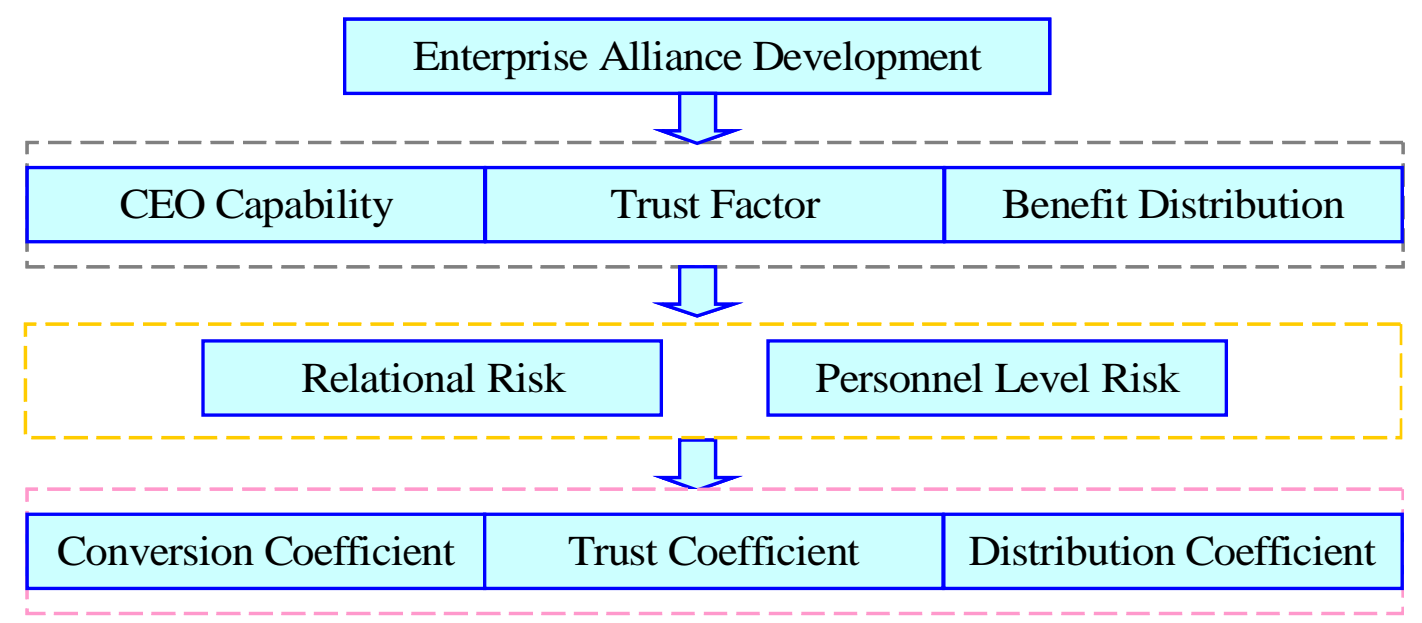

Figure.2 Model of enterprise cooperation factors under asymmetric alliance

In terms of cooperation between the partners, the high degree of trust in the cooperation of the company, as the two of the costs of the partnership are low; the cost of the cooperation is low; the cost of cooperation is low; the cost is low; that would have a huge impact on the stability of the alliance.

\section{Decision making model of alliance enterprise}

According to the method proposed by Friedman, evolution and the stability of the equilibrium point of the system by the system Jacobian matrix of local stability analysis, based on the evolution game theory and meet the det $J>0, t r J<0$ equilibrium point, for the stability and evolution of the system. In the 5 local equilibrium points, only $B(0,0)$ and $D(1,1)$ are stable, and are the two strategies, which correspond to the 3 strategies of large enterprises and small enterprises (non cooperative and non cooperative) [5]. In this evolutionary game, the analysis of the AOC region near the line, the alliance enterprise in the choice of cooperation or non cooperation, if one side by the outside opportunistic behavior, will lead to enterprise more inclined to get short-term high interests through cooperation, the alliance cooperation to regional $\mathrm{p}=0$, will eventually lead to (not cooperative, non cooperative) direction namely $\mathrm{q}=0, \mathrm{ABCO}$ convergence, reaching the evolutionary stability strategy, then the game can not continue to cooperate with the A position group, the game is not cooperative strategy, game B position group to take a non cooperative strategy.

There is a close relationship between the change of the stability of the alliance and the trust between enterprises themselves. Under the condition that the coalition environment, such as social environment, political environment, and other factors, we focus on discussing the influence of the distribution coefficient, reward and punishment coefficient, the change of the coefficient of conversion coefficient and trust coefficient on the stability and the stability of the alliance [6]. The distribution coefficient is the share of large enterprises in the alliance [7]. The small and medium enterprises in the alliance process are prone to opportunistic behavior; the fundamental reason is the unreasonable distribution of interests and the lack of trust between the two sides.

$$
\frac{d S}{d \mu}=\frac{1}{2(\Delta V-S)}\left[\frac{b V(\varpi-\theta)}{\mu^{2}}-\frac{b V(\theta-\varpi)}{(1-\mu)^{2}}\right]
$$


In view of the huge differences between different enterprises in the enterprise culture, strategic objectives, the enterprise to build the alliance cooperation must be fully prepared, must be fair and reasonable distribution of profits between enterprises, through multi-channel communication to establish long-term mutual trust and mutual relations, so as to facilitate the development of long-term cooperation between the two sides $[8,9]$. So it is necessary to establish a reasonable profit and loss distribution mechanism for the stability of the alliance [10]. The external macro environment is mainly reflected in the policy of discrimination and financing difficulties, the problem is that the problem is that small and medium enterprises in the process of cooperation in the cost of default, the proportion of total assets, the proportion of indirect costs is relatively large.

$$
\frac{d S}{d \bar{\Phi}}=\frac{1}{2}\left[\frac{-b V}{\mu(\Delta V-S)}-\frac{a V}{(1-\mu)(\Delta V-S)}\right]
$$

The more perfect the external macro environment, the higher the operation cost of small and medium enterprises, the burden of enterprises, and the development of small and medium-sized enterprises. If the small and medium-sized enterprise cooperate, the government and the intermediary organization can support them in financing, and promote the development of small and medium-sized enterprises, as shown in figure.3.

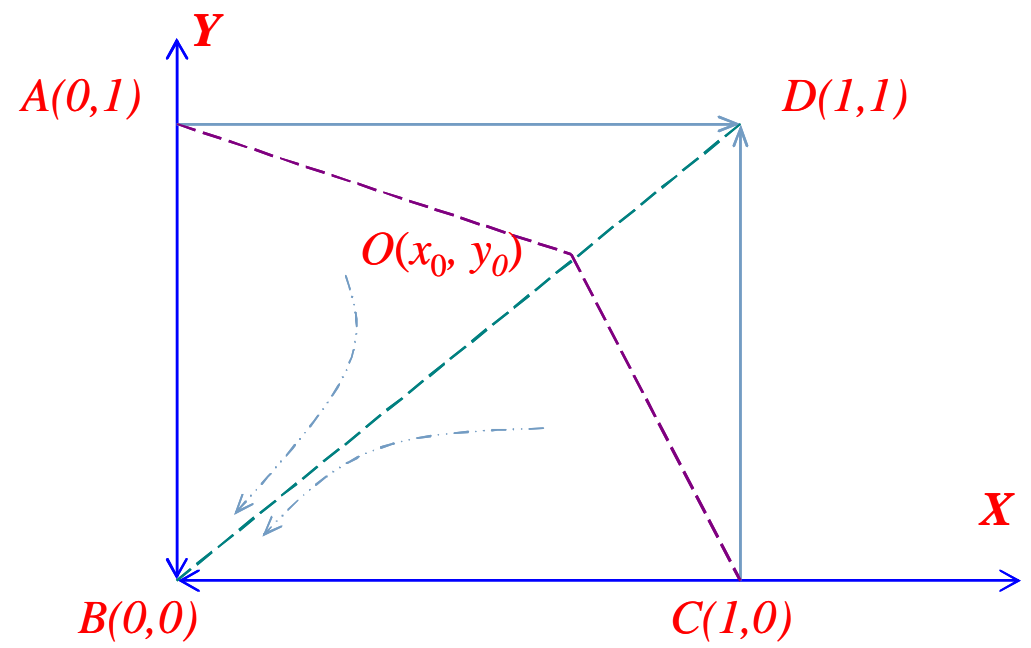

Figure.3 Evolution Evolutionary Game Party

If the long-term development of enterprises to consider the impact of factors, both sides are based on trust and cooperation in the long-term interests of the problem, the alliance cooperation to regional $\mathrm{ADCO}$, will eventually lead to (cooperation, cooperation) direction namely $\mathrm{p}=1, \mathrm{q}=1$ convergence, reaching the evolutionary stability strategy [11]. That is, the A position of the game parties to take the cooperative strategy, the game side B position groups to take a cooperative strategy, the form of asymmetric alliance will continue to develop the long-term stability of the alliance. Therefore, rewards and punishment coefficient more reward and punishment, can promote the sound development of the stability of strategic alliance, to the small and medium-sized enterprise development positive role is very big.

\section{Conclusion}

Firstly, based on the assumption of the above, the paper builds a decision game model, then uses the evolutionary game theory to analyze its equilibrium and stability, and discusses the factors that affect the stability of the alliance. Then, the paper analyzes the impact of the factors on the enterprise and the impact on the two aspects. Through the simulation analysis, the change of the internal factors of the enterprise and the evolution of the cooperative path of the large enterprises are studied, and the main influencing factors of the evolution path of the enterprise in the symbiotic state are obtained. 


\section{References}

[1] Nikolaos S. Sklavounos, Konstantinos P. Rotsios, Yannis A. Hajidimitriou, The Impact of Age, Interdependence and Perceived Risk of Opportunism on Inter-partner Trust in International Strategic Alliances, Procedia Economics and Finance, Vol.19 (2015), p.175-183

[2] Shima Mohebbi, Xueping Li, Coalitional Game Theory Approach to Modeling Suppliers' Collaboration in Supply Networks,International Journal of Production Economics, In Press, Accepted Manuscript, Available online 3 September 2015

[3] David Ahlstrom, Edward Levitas, Michael A. Hitt, M. Tina Dacin, Hong Zhu, The three faces of China: Strategic alliance partner selection in three ethnic Chinese economies, Journal of World Business, Vol.49 (2014), p.572-585

[4] Margaret A. Johnston, The sum of all fears: Stakeholder responses to sponsorship alliance risk, Tourism Management Perspectives, Vol.15 (2015), p.91-104

[5] Jung-Ho Lai, Li-Yu Chen, The valuation effect of corporate governance on stakeholder wealth: Evidence from strategic alliances, International Review of Economics \& Finance, Vol.32 (2014), p.117-131

[6] Jie Wei, Jing Zhao, Yongjian Li, Price and warranty period decisions for complementary products with horizontal firms' cooperation/noncooperation strategies, Journal of Cleaner Production, Vol.105 (2015), p.86-102

[7] Jiuh-Biing Sheu, Xiao-Qin Gao, Alliance or no alliance-Bargaining power in competing reverse supply chains, European Journal of Operational Research, Vol.233 (2014), p.313-325

[8] Natalya Kuzminykh, Pavel Zufan, Airline Alliances and their Influence on Firm Performance, Procedia Economics and Finance, Vol.12 (2014), p.329-333

[9] Son Duy Dao, Kazem Abhary, Romeo Marian, Optimisation of partner selection and collaborative transportation scheduling in Virtual Enterprises using GA, Expert Systems with Applications, Vol.41 (2014), p.6701-6717

[10] Katharina Laufs, Christian Schwens, Foreign market entry mode choice of small and medium-sized enterprises: A systematic review and future research agenda, International Business Review, Vol.23 (2014), p.1109-1126

[11] Ursula F. Ott. International Business Research and Game Theory: Looking beyond the Prisoner's Dilemma, International Business Review, Vol.22 (2013), p.480-491 